\title{
Analyzing Rich Qualitative Data to Study Pencil-Puzzle-Based Assignments in CS1 and CS2
}

\author{
Zack Butler \\ Rochester Inst. of Tech., USA \\ zjb@cs.rit.edu
}

\author{
Ivona Bezáková \\ Rochester Inst. of Tech., USA \\ ib@cs.rit.edu
}

\author{
Kimberly Fluet \\ kfluet@gmail.com
}

\begin{abstract}
Pencil puzzles (puzzles such as sudoku and many others that are designed to be solved by humans, promoting computational thinking) provide a natural context for CS1/2 assignments. In a prior work we analyzed Likert-scaled student responses and assignment/course grades to show that not only are such assignments effective but are also largely independent of gender and prior computing experience. This paper focuses on open-ended student comments, both to see if they provide additional insights about the assignments and student perceptions not apparent from the Likert-scaled responses, and to see if these comments are consistent with the results from the prior work. We surveyed over 1000 students who had used pencilpuzzle-based assignments and invited them to make open-ended comments in their survey responses. We used grounded theory to develop codes for the large volume of student survey comments, as well as for semi-structured interviews with the instructors and focus groups with student TAs. Statistical analysis of the coded comments identified several interesting relationships, such as students being appreciative of their learning even when they perceived the assignments as difficult, which were not available from the Likertscaled data. The analysis also confirmed that these assignments are largely gender- and experience-neutral. We conclude by discussing how these results and the coding process lead to improvements in assignment development and inform future research directions.
\end{abstract}

\section{CCS CONCEPTS}

- Social and professional topics $\rightarrow$ Computer science education; Computational thinking;

\section{KEYWORDS}

Introductory computer science, qualitative analysis, puzzles

\section{ACM Reference Format:}

Zack Butler, Ivona Bezáková, and Kimberly Fluet. 2018. Analyzing Rich Qualitative Data to Study Pencil-Puzzle-Based Assignments in CS1 and CS2. In Proceedings of 23rd Annual ACM Conference on Innovation and Technology in Computer Science Education (ITiCSE'18). ACM, New York, NY, USA, 6 pages. https://doi.org/10.1145/3197091.3197109

\footnotetext{
*This material is based upon work supported by the National Science Foundation under grant DUE-1245349.

Permission to make digital or hard copies of all or part of this work for personal or classroom use is granted without fee provided that copies are not made or distributed for profit or commercial advantage and that copies bear this notice and the full citation on the first page. Copyrights for components of this work owned by others than ACM must be honored. Abstracting with credit is permitted. To copy otherwise, or republish, to post on servers or to redistribute to lists, requires prior specific permission and/or a fee. Request permissions from permissions@acm.org.

ITiCSE'18, fuly 2-4, 2018, Larnaca, Cyprus

(C) 2018 Association for Computing Machinery.

ACM ISBN 978-1-4503-5707-4/18/07 ..\$15.00

https://doi.org/10.1145/3197091.3197109
}

\section{INTRODUCTION}

Developing effective educational practices and materials is an iterative process in pursuit of exceptional teaching and learning. Ideally, instructors will use evidence of learning and student feedback from each course and assignment, along with their own perceptions, to direct the development of future methodologies and assignments. For introductory computing courses there has been interest in and development of context-based learning experiences, and a number of different contexts have been explored (e.g [6, 8, 13, 21]). Various types of puzzles have been used as context for CS concepts $[4,12,15,17]$. We have previously shown pencil puzzles to be effective and largely independent of gender and prior programming experience [1], two dimensions that can impact student success at the college level. However, that study analyzed only student grades and surveys with Likert-scaled questions to determine efficacy of the pencil-puzzle-based programming assignments. In the current work, we analyze open-ended student comments to identify what specific additional insights can be gained about this type of assignment, as well as whether these comments verify the gender- and experience-neutrality previously observed. We also incorporated the reflections of experts (CS1 and CS2 instructors) as well as more experienced students (CS 1/2 teaching assistants (TAs) and lab assistants (LAs)) to investigate pencil-puzzle-based assignments across a range of perspectives [16].

We collected these perspectives specifically through 1662 openended comments (from as many completed surveys), 5 semi-structured interviews with CS1 and CS2 instructors (each 45 minutes long), and 2 focus groups, one with TAs and one with LAs (totaling 3 hours). Since these data fall into the narrative (spoken or written expression of thoughts, unconstrained in format) category of qualitative data, we sought to analyze them to retain as much richness as possible while at the same time working the data into more manageable forms. As such, we used grounded theory analysis [9] to generate coding schemes [20] for both the student survey comments and interview/focus group data, allowing us to identify relationships and draw conclusions. The coded student comments were statistically analyzed for frequency of occurrence, co-occurrence (that is, whether students who made one kind of comment were more likely to make another kind of comment), as well as for any relationships to gender and prior programming experience. This was helpful in two ways: first, by seeing what codes emerged from the coding process, we could identify broad categories of critique and appreciation of the assignments, but with deep descriptive detail. Second, by looking for statistically significant patterns within the comments, we could identify relationships between particular codes.

Finally, we examine the student perspective, instructor perspective, and TA/LA perspectives together. In particular, this information can be used to direct development of future assignments, 
improve the student surveys, generate profiles of groups of students that may benefit from or struggle with this particular context, and point to additional research directions.

\section{PENCIL PUZZLES IN INTRO CS}

As mentioned, providing a context for teaching introductory CS topics can be of great benefit. One such context is the large domain of pencil puzzles (that is, puzzles designed to be solved by humans with pencil and paper). While sudoku is a well-known example of this type of puzzle, dozens of such puzzle types are available and potentially suitable to teach computing concepts. Here we describe two particular puzzles that have been used as part of this work (assignments were given in several courses taught by instructors other than the authors), as well as how these puzzles were turned into programming assignments.

\subsection{Sample Puzzles}

Blackout Math. In a Blackout Math puzzle ${ }^{1}$, you are given a sequence of numbers and mathematical symbols, including an equals sign, and must remove (or blacken out) two of them to create a correct equation. One example with its solution is given here:

\begin{tabular}{|c|c|c|c|c|c|c|c|c|c|c|c|c|c|c|}
\hline 8 & 4 & - & I & 2 & + & 7 & $=$ & 5 & 6 & $\div$ & I & 4 & + & 9 \\
\hline 8 & & - & & 2 & + & 7 & $=$ & 5 & 6 & $\div$ & I & 4 & + & 9 \\
\hline
\end{tabular}

This puzzle was utilized as an exercise in string manipulation and parsing in CS1. Students first wrote a function that could compute the value of a simple mathematical equation (without parentheses, left-to-right order of operations). Then, they wrote a function that removed all pairs of characters from a string, essentially using a brute-force approach to solving the puzzle. A longer, project-level assignment given in a different semester also included puzzles that could have more than two characters removed from the string and required a recursive approach to generate all such sets of deleted characters to test for equality.

Lasers. Akari, which we called Lasers in our assignment, is an original puzzle published by the Japanese company $\mathrm{Nikoli}^{2}$. In this puzzle, you are given a grid with some black squares (representing walls). Some wall locations also have a number written on them. To solve the puzzle, you must place some lights (lasers) in the grid Each light illuminates the squares in its row/column, up to the next wall or edge of the grid. No laser may illuminate another, and all squares must be lit by at least one laser. Walls with numbers indicate the number of lasers immediately adjacent (horizontally or vertically) to that square. For example [19]:
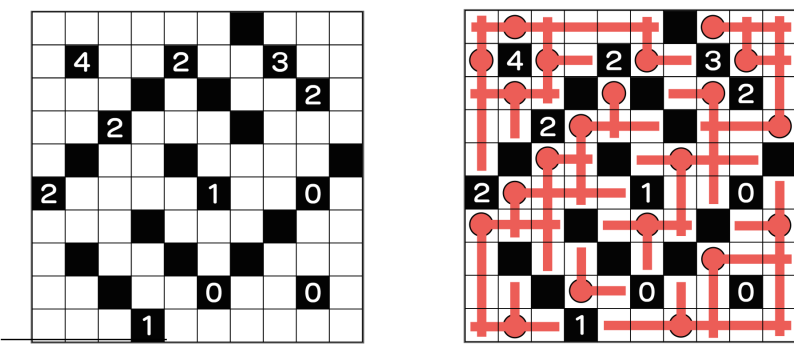

${ }^{1}$ http://www2.stetson.edu/ $/$ efriedma/published/blackout/index.html

${ }^{2}$ http://www.nikoli.com/en/puzzles/bijutsukan/rule.html
This particular puzzle was used in a two-part project in CS2. The first part of the project concentrated on solving puzzles using a backtracking algorithm. During this portion, students were encouraged to explore human-style strategies to make the solving process more efficient. For this part of the project, a simple text-based interface was used. The second part of the project involved creating a GUI for humans to solve the puzzle, with the same underlying puzzle definition, to help understand model-view-controller concepts.

\subsection{Assignment Logistics}

In our introductory courses, each assignment starts with a problem solving session which is done in groups and without electronic devices (that is, on paper or on a whiteboard). During this session, students are asked questions that lead them toward an appropriate design and/or pseudocode for the program that they will write. Once the problem solving period is over (approximately an hour), students program their individual implementations in the following hour (and subsequently at home). In these courses, instructors hold lectures and run the problem solving sessions, in addition to developing assignments and holding office hours. The TAs hold weekly recitation sessions with each section of the course, and also hold office hours during which students can seek assistance on the assignments. The LAs participate in the weekly problem solving and lab sessions, grade many of the assignments, and also hold additional office hours.

\section{RESEARCH FRAMEWORK}

Our main research direction centers around discovering new perspectives emerging from narrative data such as open-ended student comments and reflections from instructors and TAs/LAs. For example, do open-ended comments provide additional insights about pencil-puzzle-based assignments when compared with the Likertscaled survey questions used in previous work? Do these comments support the previously reported gender- and experience-neutrality, and, if yes, do they lead to new findings along these directions? As for the instructor/TA/LA reflections, we study whether this new lens sheds light on specific aspects of the assignments and student learning.

In our prior work [1], we analyzed qualitative data (Likert-scaled survey questions and demographic data) and quantitative data (assignment and final grades). However, Likert-scaled and demographic data are, by necessity, thin in detail and lacking in complexity, though they do provide opportunities to see statistically significant trends in large data sets [7]. Narrative data represent what is called 'rich' qualitative data, where "the influences of the local context are not stripped away, but are taken into account." [10]. In contrast, a Likert-scaled question simply allows no room for elucidation by the respondent ('Indicate how a pear tastes from these five choices' versus 'What does a pear taste like to you?' [5]). The challenge with descriptively rich data is to rigorously analyze the data so as to investigate relationships and draw conclusions.

\subsection{Data Collection}

All of the analyses in this paper are based on delivery of pencil puzzle assignments at RIT. Overall, we analyzed nine puzzle-based assignments across seven course offerings. Assignments were given 
in CS1, CS2, and a bridge course with similar content for underprepared incoming MS students. In each case, we administered an anonymous survey to the students after the assignment was completed. In one of the courses, we also surveyed the students after a non-puzzle-based assignment for comparison. In total we collected survey and grade data for over 1200 students (some of whom completed two surveys during their course), yielding 1662 completed surveys. In the surveys given after each assignment, in addition to asking the same Likert-scaled questions as we used in [1], we also invited the students to provide open-ended comments. We collected the same demographic data (including information about gender and programming experience, as measured by the number of programming courses taken prior to the current course), and also grade data (both for the assignment and the overall course).

The richer narrative data in our study consisted of the openended student survey comments and transcripts of the instructor interviews and TA and LA focus groups. Both the interviews and the focus groups were semi-structured, allowing us to explore relevant trains of discussion that might otherwise have been ignored in a fully structured interview [14].

\subsection{Data Analysis Methodology}

To answer the research questions, we employed a mixed methodology research approach [11] utilizing both statistical analysis and grounded theory analysis [22]. Grounded theory analysis reveals the underlying patterns in a complex data set. In Likert-scaled questions we provide only a few reasonable buckets into which we ask people to sort themselves. For narrative data, when using grounded theory methods, the data itself generates an authentic and arbitrarily rich coding scheme [20] in which a small number of descriptive codes are generated and a few of these are assigned to each item in the narrative data set. The process typically starts with line-by-line open coding (assigning a descriptor or code to a word, phrase or lines of text in the data) and the use of a constant comparative method (when assigning codes to a given portion of data, compare that data to previously coded instances of that code or category). This can be followed by examining the resulting coding, generating alternate or new codes, and recoding using the developing collection of codes [22]. Grounded theory typically results in themes that represent the complexity of the system being studied and yields a theoretical framework for use in future work [2].

\section{SURVEY COMMENT ANALYSIS}

\subsection{Initial Code Development}

We evaluated each of the 1662 comments across the nine surveys using the methodology described above. This process led to the creation of 12 emergent codes which are listed in the left-most column of Table 1. We assigned up to three codes to each comment (whichever three best described the comment). One of the more interesting codes from our initial coding was Metacognitive, which we defined as reflection on one's own learning. Since the comments about the pencil puzzle assignments were open-ended, the instances of spontaneous metacognition were noteworthy, "This lab was a massive challenge in the amount of critical thinking and understanding of python's mechanics necessary to solve it. The challenge curve for the course should be adjusted so that the transition from projects like turtle drawings to problems like these is smoother." (Comment \#184, CS1_F14_L). These initial codes were superficially descriptive, as was appropriate for this stage of analysis.

\subsection{From the Initial to the Final Coding Scheme}

Using the initial codes, each of the three researchers coded the same subset of 100 comments. We then met and systematically processed through the similarities and differences in our coding, considered codes that didn't work, those that worked well, missing codes and which codes we found particularly interesting. This process is known as triangulation, and is intended to rigorously check if and how there was inter-coder agreement among the researchers about code definitions and associated data [20]. This work yielded both new codes and more specific definitions of existing codes, listed in full in Table 1, that were more nuanced and able to represent the complexity inherent in the students' thoughts. Here we detail the evolution of the codes in each of several broad themes, and provide examples for some specific codes.

Critique and Appreciation. The initial coding scheme included the codes InstructPositive and InstructNegative for feedback about instructors and instruction, but these codes were not precise enough. For example, the InstructPositive code was used both for "Overall I enjoyed working on this project. The problem background was interesting enough and I felt I has to utilize all the skills I had learned to complete the assignment." (Comment \#1313, CS2_S16), as well as "I do appreciate the analogies in lecture, that was incredibly helpful. I strongly dislike poor grammar and typos." (Comment \#516, CS1_S16). While we were very interested in comments about learning, comments about overarching course feedback, course documents and infrastructure were less important to our current analysis, though certainly relevant for course and assignment revisions. The final codes A_Learning and C_Learning specifically address appreciation and critique of the learning experiences directly related to the pencil-puzzle-based assignment, the final codes A_Course and C_Course house overarching courserelated comments, while A_Materials and C_Materials refer to both technical and course document feedback.

As a result of triangulation, we also broadened the Puzzle code to appreciation (A_Concept) and critique (C_Concept) of the underlying puzzle concept. In doing so we articulated many more comments about the use of pencil puzzles in assignments such as, "Interesting application of binary trees. Was pretty cool to make a program that solves blackout math.” (Comment \#594, CS1_S16).

Enjoy and Disapprove. From the initial coding there were quite clearly many positive and many negative comments. However, during triangulation we explored expressions of vehement emotion as they occurred separately or with comments of other content. We decided to redefine the codes Positive and Negative to Enjoy and Disapprove. Enjoy and Disapprove were also more limited in scope to data fragments that were expressions of strong emotion. The following quote was coded, using the final codes as Enjoy, A_Learning and C_Materials, "This project most definitely taught me the importance of testing my functions as I go along. Especially when it came to handling the compute function that was the most stressful, but once it worked correctly, it was very exciting to see that my coding skills are improving. The writeup was written a bit 
Table 1: Initial and final codes for open-ended student survey comments

\begin{tabular}{|c|c|c|}
\hline Initial Codes & Final Codes & Final Definition \\
\hline Positive & Enjoy & $\begin{array}{l}\text { positive, vehemently positive, explicit statement of enjoyment, 'I loved it', has an emotional } \\
\text { quality (not neutral fact) }\end{array}$ \\
\hline Negative & Disapprove & $\begin{array}{l}\text { negative, vehemently negative, Disapprove- grumpy face, disliked, frustrated, also has an } \\
\text { emotional quality 'I hated it' (not neutral fact) }\end{array}$ \\
\hline Mixed & [None] & \\
\hline Puzzle & $\begin{array}{l}\text { A_Concept } \\
\text { C_Concept }\end{array}$ & $\begin{array}{l}\text { appreciation of the lab/project concept specifically related to pencil puzzles } \\
\text { critique of the lab/project concept specifically related to pencil puzzles }\end{array}$ \\
\hline [None] & $\begin{array}{l}\text { A_Learning } \\
\text { C_Learning }\end{array}$ & $\begin{array}{l}\text { appreciation of their learning experience } \\
\text { critique of their learning experience }\end{array}$ \\
\hline $\begin{array}{l}\text { TechPositive } \\
\text { TechNegative }\end{array}$ & $\begin{array}{l}\text { A_Materials } \\
\text { C_Materials }\end{array}$ & $\begin{array}{l}\text { appreciation of the materials, infrastructure, writeups, technical choices in lab/project } \\
\text { critique of the materials, infrastructure, writeups, technical choices in lab/project }\end{array}$ \\
\hline InstructPositive & A_Course & $\begin{array}{l}\text { appreciation of the course, the timing of assignments, the alignment of lecture with lab/project, } \\
\text { the instructors teaching choices, extending beyond the lab/project in question }\end{array}$ \\
\hline InstructNegative & C_Course & $\begin{array}{l}\text { critique of the course, the timing of assignments, the misalignment of lecture with lab/project, } \\
\text { the instructors teaching choices, extending beyond the lab/project in question }\end{array}$ \\
\hline [None] & $\begin{array}{l}\text { Difficulty_fair } \\
\text { Difficulty_easy } \\
\text { Difficulty_hard }\end{array}$ & $\begin{array}{l}\text { the lab/project was fair } \\
\text { the lab/project was easy } \\
\text { the lab/project was hard, frustrating, confusing }\end{array}$ \\
\hline Metacognitive & $\begin{array}{l}\text { Metacognition } \\
\text { R_Resources } \\
\text { R_Time } \\
\text { R_Experience }\end{array}$ & $\begin{array}{l}\text { thinking about their thinking } \\
\text { reflection on use/not use of resources, eg. help center, other students, instructor } \\
\text { reflection on time spent or time management under their own control } \\
\text { reflection on role of experience/prior learning in completing assignment }\end{array}$ \\
\hline [None $]$ & Don't_understand & I don’t get it, I don't understand, hard to understand, I don’t even know what I don’t know \\
\hline Unintelligible & Irrelevant & $\begin{array}{l}\text { gobbledygook or statement about something unrelated to the course; possibly also a statement } \\
\text { of fact that has no relevance to the course, e.g. The sky is blue }\end{array}$ \\
\hline Null & Null & no answer or no or nope \\
\hline
\end{tabular}

poorly. Some parts were incredibly vague while others were very detailed. Overall, I was very pleased with myself once everything worked properly!" (Comment \#619, CS1_S16)

Difficulty. Each member of the research team struggled with sorting statements about difficulty into the initial codes. There were many comments that referenced difficulty. In some instances it was not clear if comments about difficulty included a value judgment (good or bad), while other comments about difficulty included critical or appreciative statements. Thus, the stand-alone codes Difficulty_hard and Difficulty_easy were created. For example, the comment "Even though it was tough, finishing the lab in what I believe to be a correct manor was very, very satisfying." (Comment \#383, CS1_F14) was coded both Difficulty_hard and Enjoy. In addition, after all the data were recoded using the final coding scheme, we encountered a set of data that asserted the fairness of the pencil puzzle assignments. And so, Difficulty_fair was added to the coding scheme.

Reflection and Metacognition. In the initial coding scheme, Metacognitive captured thinking about one's own thinking and incidentally also captured moments of reflection, careful consideration and analysis of ideas, events, and/or one's own experiences. After triangulation we planned to continue using the Metacognitive code. However, in the midst of recoding the comments using the final coding scheme, we encountered many instances which were not strictly examples of metacognition nor did they fit elsewhere. From an examination of the literature on metacognition, we more precisely distinguished metacognition as a specific kind of reflection on one's own thinking processes and created additional codes that better distinguished reflective thinking [18]. The following comment was subsequently coded as Metacognition and as C_Materials, "I thought it was a decent project/lab. What I had trouble most with, was understanding the directions out of everything. I understand the lab conceptually, however, there is still that barrier between understanding how to solve the problem and understanding how to code." (Comment \#586, CS1_S16) while other reflective comments were coded as reflection about resources, reflection on experience level and/or reflection on time, e.g. "I should have started earlier." (Comment \#572 CS1_S16) which was coded as R_Time.

Don't Understand. Lastly, with most of the data coded using the final coding scheme, a small number of comments refused to be categorized into the existing codes. When examined together, we found these were linked to comments about frustration, but lacked a specific focus for the frustration. Taking the code name from the comments themselves we created the code Don' $t$ _understand. In each instance that we coded Don' $t$ _understand, the individual is able to share no more specificity than that they don't understand. If a comment referred to not understanding the course materials, e.g. 'I didn't understand the instructions', it was coded specifically as critique of the materials, or if a comment referred to not understanding that week's content, such as backtracking, then it was 
coded as critique of learning. Thus, the remaining instances that were coded Don' $t$ _understand were those where the individual has such cognitive overload [3] that they don't even know what they don't understand. "It can feel like every week is forcibly pushing me onto the next unit while the last unit was a struggle to the end, resulting in a vague understanding of how the concepts work without the ability to recreate the lessons effectively." (Comment \#504, CS1_SP16_L). These are the students who truly struggled.

\subsection{Statistical Findings}

Once the comments had been recoded using the final codes (using as many codes as applicable per comment), we looked for statistically significant relationships between the various codes - that is, whether students who wrote one particular type of comment also wrote another specific type. We also looked for relationships between comment codes and gender and experience level. The relationships were investigated using ANOVA computations.

Through this process, we found several intriguing relationships. For example, in a number of cases, there was a statistically significant relationship between comments about the difficulty of the lab (Difficulty_hard) and comments expressing an appreciation for the learning experiences (A_Learning). For the earliest assignment, the Blackout Math Lab in CS1, this relationship was significant with $\mathrm{p}=.002$. This was particularly surprising in that many students expressed frustration with the assignment (in the Likert-scaled questions), leading us to a revision of the assignment for future terms, yet many of these same students also had appreciated the assignment. The same positive relationship between Difficulty_hard and A_Learning also appeared in the revised Blackout Math assignment (now a project) in a later offering of CS1 $(p=.012)$. For comparative purposes we ran the same analysis for a non-puzzle-based assignment during this same later CS1 course. Here, we found the opposite relationship: there was a statistically significant relationship between mentioning the difficulty as hard (Difficulty_Hard) and both disapproval of the assignment (Disapprove, $\mathrm{p}=.027$ ) and critique of the learning experience (C_Learning, $\mathrm{p}=.000$ ). These results indicate that perception of difficulty of an assignment is neither inherently good or bad, but that for these pencil puzzle assignments, while perceived as difficult, students do have an appreciation for the learning experience.

We were also encouraged to find that this positive relationship between Difficulty_hard and A_Learning was present in the Lasers project in CS2 $(\mathrm{p}=.001)$ and another puzzle-based lab in the same course $(\mathrm{p}=.012)$. In the Lasers project, we also found a strong relationship ( $\mathrm{p}=.029)$ between mentioning that the assignment was hard (Difficulty_hard) and that they enjoyed it (Enjoy).

We also analyzed the comment codes for relationships to gender and experience level. Our findings are in line with those previously reported [1], supporting the experience- and gender-neutrality of the pencil puzzle context. One particular exception was found in the CS2 Lasers project. Despite the fact that the Likert responses on the same survey showed no statistical relationship to gender, we found statistically significant relationships between gender and the comment codes Difficulty_hard, Difficulty_easy, and Don' t_understand. In particular, women were more likely to comment that they found the project difficult, that they found it easy, and that they didn't understand. This implies that the narrative data can reveal complexity that the simpler Likert data cannot.

It is important as well to note that the codes that had the most statistical meaning were a result of triangulating and revising our coding scheme. In doing so we created a framework that allowed us to better describe our data. These statistically significant relationships give us a more rich understanding of students' experiences of pencil-puzzle-based assignments that simply could not be measured with the original Likert-scale survey questions. These understandings will certainly guide future investigations.

\section{INTERVIEW AND FOCUS GROUP ANALYSIS}

As previously mentioned, we were interested in collecting multiple perspectives about the efficacy and experiences of the pencil puzzle assignments. We conducted interviews and focus groups with the course instructors and the student TAs/LAs to learn about their perceptions of the assignments and to get a density of data relating to our research questions [2]. The interviews and focus groups were audio-recorded with permission and transcribed verbatim.

Analysis of the transcribed data was performed in a similar fashion to the open-ended survey comments, using grounded theory to extract a framework from the data. The data was coded through line-by-line, open-coding and constant comparative methods, with one cycle of code development. As a result of the coding process, we articulated several codes and related emergent themes. However, as might be expected, given the participants were CS faculty and advanced undergraduate and graduate students, the codes did not always match the concerns (or appreciations) of the students, and as such they cannot be directly correlated with the codes in Table 1. Here we discuss the major themes and their associated codes.

Thriving. One theme that emerged was the various ways in which students thrived in the pencil puzzle context. Strategy, Creativity, Competition, and Pride were recurring codes from both the Instructors and the TAs/LAs. As an example, the following comment was coded as Pride: "so it provides a real sense of accomplishment when they can actually show off to non-CS people and have them think 'ok this is cool' 'cause it's more of a puzzle-type thing and not like 'oh let's create this database'." (TA_FocusGroup).

Puzzle People. Another interesting theme was the question of whether there are Puzzle_people and Non-puzzle_people. Some instructors and TAs/LAs saw their students as being puzzle people and therefore inherently engaged by these assignments. Others wondered if the students who didn't do well or like the assignments were simply not puzzle people, for example, "Um, so when it comes to puzzles I feel like it works well in large part just because the type of people that are taking these classes, to a large extent, like puzzles, they like puzzles. That's just who, who they are, how their brains are wired. But that's not everybody. . when they're on their own on a Saturday and they've got some free time. Sitting down and cranking out puzzles is_not_in their top 50 things they want to do. Um, (pause) so that said, um sometimes a puzzle is still really good for teaching the concept, whether it's a data structure or an algorithm" (Instructor_1). We are left to consider whether proclivity for puzzles is a determining factor, or not, in students' success with pencil-puzzle-based assignments. However, we also note that this type of question could be asked for any particular context, and in 
fact, we did not get a single comment from a student describing themselves as a non-puzzle person. Nonetheless, in the future, it may be valuable to revise the survey to target this idea.

Struggling. One common area of concern for instructors as well as the TAs and LAs was that of helping Struggling students. In particular, the instructors and (especially) TAs and LAs found cognitive overload [3] to be a serious challenge, "Kind of what happens at the other end of the spectrum, like students who come in and they tend to struggle with almost all of the labs and now they are facing a puzzle lab, is it, are they struggling equally badly as before or are they struggling more? How does it effect them?" (LA_FocusGroup). In some cases, this might be due in part to the materials and/or concepts being hard to understand, particularly as a novice, "so I don't know how much of that actually speaks to the puzzle aspect and how much of it just is that grouping of students that have a really hard time with the programming part of things." (TA_FocusGroup).

However, even when the instructors foreground these issues in course/material design, as we have, some students can still feel completely lost and overwhelmed, as seen in the student comment data (notably in the comments coded Don' $t$ _Understand). It can be hard to deduce whether a student is in fact struggling with the entire concept, or simply a particular implementation. Perhaps the comment codes of C_Materials and C_Course can get at some of these problems, but the student may be unable to even formulate their concerns. Perhaps the TAs and LAs may also need more training as to how to drill down to the underlying barriers to understanding that a particular student may have.

\section{DISCUSSION}

We applied grounded theory to qualitatively analyze open-ended comments about pencil-puzzle-based assignments in large introductory CS courses. We have demonstrated that it is plausible to do so, and in fact we learned interesting lessons about these assignments as described in Sec. 4.3, notably that students often appreciate their learning experience even when they perceive the assignment as difficult. Our analysis confirmed that pencil-puzzle-based assignments are largely gender- and experience-neutral. We also used grounded theory to analyze instructor interviews and TA/LA focus groups as explored in Sec. 5.

Future puzzle-based assignments will benefit from both the openended comment analysis and interview/focus group analysis. For example, instructors should not necessarily shy away from difficult assignments, but it is also important to provide additional supports for struggling students. Such supports could include training the TAs and LAs to assist students through conceptual barriers to learning the CS concepts.

Both analyses will also impact our survey design. We anticipate creating Likert-scaled survey questions that better capture appreciation for the learning experience in spite of (or perhaps because of) the difficulty of the assignment. We will also add prompts for more demographic descriptors, such as academic major, and to identify additional under-represented students, as well as survey questions about problem solving style and puzzle proclivity.

The open-ended comment analysis and interview/focus group analysis also have direct bearing on future research directions. We wish to look more closely at those who thrive and those who struggle to understand. To do so we plan to study the ability of students to exhibit transfer of understanding gained from the pencilpuzzle-based assignment to a novel scenario.

In summary, using grounded theory methodology, we rigorously and systematically analyzed all comments, finding relationships among the data, and helping us to view the comments more objectively than only reacting to specific memorable comments. This methodology can also be applied in other CS contexts, for other assignments, or open-ended comments on end-of-term course evaluations, etc. While this is more time consuming than using a simple Likert scale, it allows the students to express themselves freely, and their responses can be formally and meaningfully analyzed. We do not foresee such an analysis to be done frequently, rather we expect to do so occasionally to see if current survey instruments should be adjusted. As such, this process can form an important part of continuous improvement as we seek to make pencil-puzzle-based assignments and other CS contexts more effective for all students.

\section{REFERENCES}

[1] Z. Butler, I. Bezáková, and K. Fluet. 2017. Pencil Puzzles for Introductory Computer Science: an Experience- and Gender-Neutral Context. In SIGCSE '17. ACM, 93-98.

[2] K. Charmaz. 2006. Constructing Grounded Theory: A Practical Guide Through Qualitative Analysis. SAGE Publications, London.

[3] M. Chinnappan and P. A. Chandler. 2010. Managing cognitive load in the mathematics classroom. Australian Mathematics Teacher 66, 1 (2010), 5-11.

[4] John Cigas and Wen-Jung Hsin. 2005. Teaching Proofs and Algorithms in Discrete Mathematics with Online Visual Logic Puzzles. 7. Educ. Resour. Comput. 5, 2, Article 2 (June 2005).

[5] B. Silberling (Director). 1998. City of Angels. United States: Warner Bros.. (1998).

[6] Zachary Dodds, Ran Libeskind-Hadas, and Eliot Bush. 2010. When CS 1 is biology 1: crossdisciplinary collaboration as CS context. In ITiCSE '10. ACM, 219-223.

[7] A. Fink and J. Kosecoff. 1998. How to conduct surveys: A step-by-step guide (2 ed.). SAGE Publications, London.

[8] Samantha L. Finkelstein, Eve Powell, Andrew Hicks, Katelyn Doran, Sandhya Rani Charugulla, and Tiffany Barnes. 2010. SNAG: using social networking games to increase student retention in computer science. In ITiCSE '10. ACM, 142-146.

[9] B.G. Glaser and A. L. Strauss. 1973. The discovery of grounded theory: Strategies for qualitative research. Aldine Publishing Company, Chicago.

[10] A. M. Huberman and M. B. Miles. 1994. Data Management and Analysis Methods. In Handbook of Qualitative Research, N. K. Denzin and Y. S. Lincoln (Eds.). SAGE Publications, London, 428-444.

[11] R. B. Johnson and A. J. Onwuegbuzie. 2004. Mixed Methods Research: A Research Paradigm Whose Time Has Come. Educational Researcher 33, 7 (2004), 14-26.

[12] Anany Levitin. 2005. Analyze That: Puzzles and Analysis of Algorithms. In SIGCSE '05. ACM, 171-175.

[13] Stefanie A. Markham and K. N. King. 2010. Using personal robots in CS1: experiences, outcomes, and attitudinal influences. In ITiCSE '10. ACM, 204-208.

[14] C. A. Mertler. 2006. Action research: teachers as researchers in the classroom. SAGE Publications, London.

[15] Zbigniew Michalewicz and Matthew Michalewicz. 2008. Puzzle-Based Learning: An introduction to critical thinking, mathematics, and problem solving. Hybrid Publishers.

[16] M. A. Muecke. 1994. On the Evaluation of Ethnographies. In Critical Issues in Qualitative Research Methods, J. M. Morse (Ed.). SAGE Publications, 187-209.

[17] Behrooz Parhami. 2009. Puzzling Problems in Computer Engineering. Computer 42, 3 (March 2009), 26-29.

[18] C. Rogers. 2002. Defining reflection: Another look at John Dewey and reflective thinking. Teachers College Record 104, 4 (2002), 842-866.

[19] Nobuyuki Sakamoto. 2006. Akari Sample Problem 3. http://www. nikoli.com/en/puzzles/bi jutsukan/. (2006).

[20] J. Saldaña. 2013. The coding manual for qualitative researchers. SAGE Publications, London.

[21] Beth Simon, Päivi Kinnunen, Leo Porter, and Dov Zazkis. 2010. Experience report: CS1 for majors with media computation. In ITiCSE '10. ACM, 214-218.

[22] A. L. Strauss and J. Corbin. 1990. Basics of qualitative research: Grounded theory procedures and techniques. SAGE Publications, Newbury Park, CA. 